\title{
Topical anaesthetic applied at triage reduced treatment time in children presenting to the emergency department with minor lacerations
}

Priestley S, Kelly AM, Chow L, et al. Application of topical local anesthetic at triage reduces treatment time for children with lacerations: a randomized controlled trial. Ann Emerg Med 2003;42:34-40.

$Q$ In children with simple lacerations, does application of topical local anaesthesia at triage reduce total treatment time in the emergency department (ED)?

\section{METHODS}

$\square$

Design: randomised controlled trial.

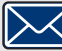

Allocation: concealed.

Blinding: blinded (patients and physicians deciding on the method of wound repair and need for sedation or anaesthesia).

Follow up period: to discharge

Setting: an urban paediatric ED in Melbourne, Victoria, Australia.

Patients: 183 children who were 1-10 years of age (mean age 4 $y, 61 \%$ boys) $^{*}$ and had simple lacerations that might require wound repair under local anaesthesia. Exclusion criteria: wounds involving the digits, ears, penis, nose, or mucous membranes; wounds close to the eye; deep wounds involving bone, cartilage, tendon, or muscles; wounds $>6$ hours old; anaesthetic application or infiltration of wound before ED presentation; and previous reaction to local anaesthesia.

$\mathbf{R}_{\mathbf{X}}$

Interventions: ALA solution (adrenaline 1:1000, lignocaine 4\%, and amethocaine $0.5 \%)(n=94)$ or placebo solution (adrenaline $1: 1000)(n=89)$ applied at triage. After application of the solution, a physician decided on the method of wound repair (Steristrips, glue, or sutures) and if [further] anaesthesia or sedation was required.

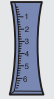

Outcomes: total treatment time ( $\mathrm{min}$ from triage to discharge) and requirement of sedation.

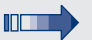

Patient follow up: $88 \%$.

*Based on 161 children included in analysis.

\section{MAIN RESULTS}

Most wounds were on the head or face. 84 wounds were glued, 65 were sutured, 6 were repaired with Steristrips, and 6 were not treated with a specific closure. Children in the ALA group had a shorter mean treatment time than those in the placebo group (77 v $108 \mathrm{~min}$, effect size $31 \mathrm{~min}, 95 \%$ CI 15 to 47). The ALA and placebo groups did not differ for sedation rates ( $12 \% v 17 \%$, effect size $5 \%$, CI -16 to 6 ).

For correspondence: Professor $A$ $M$ Kelly, Department of Emergency Medicine, Western Hospital, Footscray, Victoria, 3011, Australia. AnneMaree.Kelly@wh.org.au

Source of funding: Emergency Medicine Foundation (Australia).

\section{CONCLUSION}

In children presenting to the emergency department with simple lacerations, application of a topical anaesthetic at triage reduced treatment time compared with placebo, but had no effect on need for sedation.

\section{Commentary}

$\mathrm{n}$ addition to reducing the overall time spent in an ED, topical anaesthetic has other advantages over local infiltration anaesthetic for management of lacerations in children, ${ }^{1}$ such as reducing the associated trauma (both psychological and physical) for the child and family. The paediatric ethic of care dictates that children's distress be appropriately managed to ensure positive psychological outcomes. Such consideration should be inherent in any investigation of quality improvement strategies in paediatric EDs.

The study by Priestley et al was well designed, with blinding continuing until the wound was fully assessed and decisions were made on the method of closure and need for sedation. Although the authors found that application of topical anaesthetic reduced total treatment time, waiting time to physician treatment was not reduced.

Implementation of this strategy in paediatric EDs will require that triage nurses receive appropriate training in the assessment of lacerations and application of local anaesthetic. Availability of topical anaesthetic solutions may depend on local manufacture. Wide applicability of this strategy would be limited by the need to exclude wounds to extremities such as a finger, toe, penis, nose, or pinna. Ongoing training of nursing staff in the management of simple lacerations is another strategy that could reduce waiting time to treatment and enhance emergency nursing skills and satisfaction. Factors that may increase total treatment time, such as high acuity levels and low staff skill mix in an ED, should be considered before implementing this strategy. Additional strategies for managing child and family distress (eg, involvement of play specialists and tools that measure children's anxiety and pain) and the child's previous health experiences should also be considered.

Sarah Little, RGON, MA (App) Starship Children's Hospital Jane Brosnahan, RCpN, BN, MCNA (NZ) Centre for Evidence Based Nursing Aotearoa Auckland District Health Board Auckland, New Zealand

1 Schilling CG, Bank DE, Borchert BA, et al. Tetracaine, epinephrine (adrenalin), and cocaine (TAC) versus lidocaine, epinephrine, and tetracaine (LET) for anesthesia of lacerations in children. Ann Emerg Med 1995;25:203-8. 